\title{
An Enhanced Method for Dynamic Characterization of High-Power LEDs for Visible Light Communication Applications
}

\author{
Juan S. Betancourt Perlaza ${ }^{1, *,+} \oplus^{+}$, Juan C. Torres Zafra ${ }^{1,+}+\mathbb{C}$, Máximo Morales Céspedes ${ }^{2}$, Iñaki Martínez-Sarriegui ${ }^{3}$, \\ Carlos I. del Valle ${ }^{3}$ (i) and José M. Sánchez Pena ${ }^{1}$ (i) \\ 1 Displays and Photonic Applications Group, Electronic Technology Department, Carlos III University, \\ Butarque 15, 28911 Leganés, Spain; jctzafra@ing.uc3m.es (J.C.T.Z.); jmpena@ing.uc3m.es (J.M.S.P.) \\ 2 Signal Theory and Communications, Carlos III University, Butarque 15, 28911 Leganés, Spain; \\ mmcesped@ing.uc3m.es \\ 3 Optiva Media, Edif. Europa II, Calle Musgo 2, 28023 Madrid, Spain; \\ inaki.martinez@optivamedia.com (I.M.-S.); carlosivan.delvalle@optivamedia.com (C.I.d.V.) \\ * Correspondence: jbetanco@ing.uc3m.es \\ + These authors contributed equally to this work.
}

check for updates

Citation: Betancourt Perlaza, J.S.; Torres Zafra, J.C.; Morales Céspedes, M.; Martínez-Sarriegui, I.; del Valle,

C.I.; Sánchez Pena, J.M. An Enhanced Method for Dynamic

Characterization of High-Power

LEDs for Visible Light

Communication Applications.

Electronics 2022, 11, 292. https://

doi.org/10.3390/electronics11030292

Academic Editors: Konstantinos

Peppas, Hector E. Nistazakis and

Andreas D. Tsigopoulos

Received: 21 December 2021

Accepted: 16 January 2022

Published: 18 January 2022

Publisher's Note: MDPI stays neutral with regard to jurisdictional claims in published maps and institutional affiliations.

Copyright: (C) 2022 by the authors. Licensee MDPI, Basel, Switzerland. This article is an open access article distributed under the terms and conditions of the Creative Commons Attribution (CC BY) license (https:// creativecommons.org/licenses/by/ $4.0 /)$.

\begin{abstract}
Visible light communications (VLC) have been proposed for several applications beyond the traditional indoor scenarios, from vehicular to underwater communications. The common element in all these applications is the use of light-emitting diodes (LEDs) in which the forward current that flows through each LED plays a major role. Therefore, knowing the electrical equivalent of the LEDs is a useful tool for the proper design of the VLC systems. Currently, some measurement instruments exist, such as the LCR (inductance, capacitance, and resistance) meters or impedance analyzers to characterize the main parameters of the LEDs. However, these instruments and measurement procedures are subject to satisfying some requirements, such as a minimum value of the input impedance or the maximum forward current. In this work, the LED LXHL-BW02 is used to obtain its equivalent circuit, using different measurement methods and traditional methods of measurement with our proposed method. The equivalent model is implemented on the simulation tool LTSPICE. Our alternative method can be used for determining the electrical equivalent circuit of LEDs subject to high current variations at very high frequencies, in the $\mathrm{MHz}$ range, i.e., in an operating range for VLC applications.
\end{abstract}

Keywords: light emitting diode; equivalent circuit; visible light communications; modulation bandwidth

\section{Introduction}

In the mid 1990s, highly efficient blue LEDs were proposed for generating white light lamps [1,2]. To do that, a thin phosphorous layer is added to blue LEDs. Then, the electrical current generates blue light, and that blue light at the same time generates a yellow light by stimulating the phosphorous layer. It is important to point out that all the blue light generated is not absorbed (reconverted) by the phosphorous layer. Therefore, the resulting light is a combination of two colors, blue and yellow, and, for the human eye, this combination of colors with the right intensity is seen as white light. In 2006, a novel way to create white LEDs was proposed by Narukawa et al. By the use of gallium-indium nitride (InGaN), they achieved efficiencies of up to $174 \mathrm{~lm} / \mathrm{W}$, using significantly low currents (around $2 \mathrm{~mA}$ ). This new approach can be seen as a breakthrough to substitute the conventional white bulbs with LED-based lamps [3].

In the beginning, white LED lamps were used basically for providing illumination. However, as the continuous increase in the demand for connectivity has led to a search to exploit new parts of the electromagnetic spectrum, the research efforts are focused on modulating the drive and forward current of the LEDs for transmitting information while satisfying the illumination requirements in both indoor and outdoor environments. This 
is known as VLC or light fidelity (Li-Fi) when integrating the communication link in the IP/TCP network [4]. VLC is proposed as a means of moving part of the data traffic from the overwhelmed radio frequency (RF) spectrum, which is typically between $300 \mathrm{MHz}$ and $30 \mathrm{GHz}$, to the optical domain based on the light-emitting diode (LED) technology [5,6]. In this sense, the optical domain offers a huge and unlicensed bandwidth in which several signal processing techniques can be applied in order to overcome some limitations, such as the need for line of sight (LoS) or the interference because of transmission from neighboring light points [7]. This technology was proposed for several scenarios and applications, e.g., in environments where RF transmission is discouraged or even not allowed, such as oil and gas plants, the mining industry or hospitals [8-10], and in alternative geolocation and positioning schemes inside buildings or industrial plants, where satellite geolocation fails [11-13]. However, in a practical implementation, a small portion of this part of the spectrum can be exploited since LEDs currently are employed for illumination only and, consequently, are characterized for illumination purposes but not for data transmission.

Most of the works in the state-of-the-art about VLC are focused on achieving high data rates by using novel modulation schemes and solutions so that the achieved performance can be compared with commercial RF schemes, such as Wi-Fi or the fourth- and fifthgeneration (4G and 5G) cellular systems [14-17]. In this sense, it is worth recalling that VLC is still in the development phase, and therefore, several issues must still be solved. Indeed, the implementation of VLC prototypes and testbeds results in the need for enhancing the bandwidth of the LEDs since this element is usually the bottleneck in order to use higher modulation rates $[18,19]$.

Focusing on future sixth generation (6G) communications, VLC is considered a key technology for several applications; from indoor high-speed access to ultra-reliable lowspeed communications for positioning/navigation or the Internet of Things [20]. In [21], the extremely large bandwidth of the optical domain is pointed out as the main advantage of VLC for 6G. However, the modulation bandwidth of LED transmitters is typically the bottleneck for exploiting this huge available bandwidth in the optical spectrum. Furthermore, beyond the knowledge of the optical spectrum, it is necessary to determine the channel response of the optical transmitter in order to design the pre-distortion model or equalizer that exploits the whole modulation bandwidth [22].

Nowadays, VLC is halfway from the purely theoretical concept to typical commercial and industrial applications. In this sense, there are several works that obtain high data rates in laboratory environments. For instance, in [14], it is demonstrated that a data rate of up to $3 \mathrm{~Gb} / \mathrm{s}$ is achievable using a specific $\mu$-LED for this experiment. Moving to the use of commercial LEDs, in [23], the authors achieved a data rate of up to $40 \mathrm{Mb} / \mathrm{s}$ in a laboratory environment. However, according to the last report of the European Commission about the LED lighting maker [24], the development of VLC within the concept of "Lighting 2.0" requires the exploitation of commercial LEDs in order to achieve an efficient and sustainable technology.

In this context, it is necessary to determine the equivalent circuit model of the LEDs beyond the illumination operating point, i.e., when a high-frequency signal modulates the input current of the LED. Recently, in [25], the equivalent circuit for a commercial LED was obtained. However, the results obtained in this work are limited to a current below $35.8 \mathrm{~mA}$, which hampers its implementation for high-power LEDs. Therefore, novel measuring methodologies for determining the equivalent circuit of the LED transmitters at medium/high frequencies are mandatory for the deployment of VLC applications using medium/high power LEDs, i.e., assuming an operating current greater than $50 \mathrm{~mA}$.

In this work, we analyze the electrical behavior of illumination LEDs subject to high current variations at very high frequency, in the range of $\mathrm{MHz}$, as occurs in VLC applications. The dynamic response of the LEDs can be defined by the combination of passive electronic elements so that the electronic equivalent represents the physical behavior of the LEDs. Notice that the combination of these components corresponds to complex impedance measurements. First, we analyze the traditional methods for characterizing 
the equivalent circuit of the LEDs. The limitations of these methods when applied to LED lamps oriented to VLC applications are highlighted. After that, we propose an alternative methodology for determining the equivalent circuit of the LED lamps employed for VLC. Finally, the obtained results are validated through experimental simulations using LTSPICE software.

The remainder of this paper is organized as follows. In Section 2, the dynamic characterization of the LED is presented. The use of traditional measurement tools is analyzed in Section 2.1. After that, an alternative methodology for determining the equivalent circuit model is proposed in Section 2.2. The simulation results and corresponding equivalent circuits are depicted and analyzed in Section 3. Finally, Section 4 provides concluding remarks.

\section{Dynamic Characterization of the LEDs}

To understand the dynamic behavior of illumination LEDs is indispensable to know the equivalent circuit that models it. The simplified equivalent LED circuit comprises two mechanisms that affect directly the electrical behavior at high frequencies. These two mechanisms can be modeled as capacitance, known as the diffusion, and the barrier (or depletion) capacitance, which are in fact non-linear electronic elements. The first one is related to the atom ions' electronic charge. The second capacitance is related to the excess of minority charged carriers injected on direct polarization conditions [26]. As the capacitance types are in parallel and cannot be measured independently, both are resumed in an equivalent capacitance, from now on, the joint capacitance. Thus, the equivalent circuit in AC of a LED is as shown in Figure 1. Notice that the joint capacitance, i.e., the sum of the barrier and diffusion capacitance, determines the response of the LED in time and frequency. Parallel to the joint capacitance, the equivalent circuit of a LED comprises a resistance in parallel denoted by $R_{p}$, whose value changes with the voltage variations and series resistance $\left(R_{S}\right)$ given by the PN junction's neutral region of the LED.

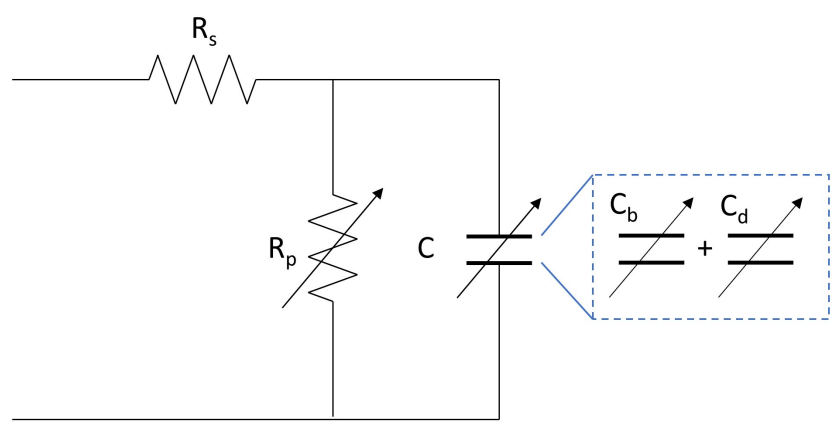

Figure 1. Equivalent circuit in AC.

\subsection{Impedance Measurement of the LED Using an Impedance Analyzer}

As mentioned earlier, to model the dynamic behavior of the LED, it is needed to obtain the equivalent electric circuit depicted in Figure 1. The values of the passive components that compose the equivalent circuit can be obtained by performing complex impedance measurements. These measurements (magnitude and phase) were obtained at first by using an impedance analyzer, from now on, the SOLARTRON 1260 analyzer (Solartron Instruments, Hampshire, UK). The SOLARTRON 1260 can perform measurements from $0.1 \mathrm{MHz}$ to $32 \mathrm{MHz}$ and can supply a DC bias up to $\pm 40.95 \mathrm{~V}$ for voltage mode and $\pm 100 \mathrm{~mA}$ for the current mode. The white LED used to perform all the measurements is the LXHL-BW02 (Figure 2) fabricated by Philips Lumileds LUXEON in San Jose (USA), Penang (Malaysia) and Singapore [27]. 


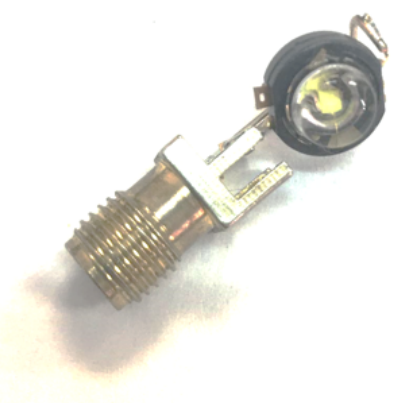

Figure 2. Measured LED LXHL-BW02.

At first, a frequency swipe from $100 \mathrm{~Hz}$ to $5 \mathrm{MHz}$ with a $10 \mathrm{~m} V_{R M S}$ sinusoidal signal and 0 DC bias is performed. Following the bode diagram in Figure 3, it can be seen that the LED behaves nearly as a full capacity load, i.e., phase equal to $90^{\circ}$. The resulting equivalent circuit has a resistor of $0.85 \Omega$ in series with a capacitance of $0.95 \mathrm{nF}$. In this configuration, the impact of $R_{p}$ in the measurement is negligible, as shown in Figure 4.

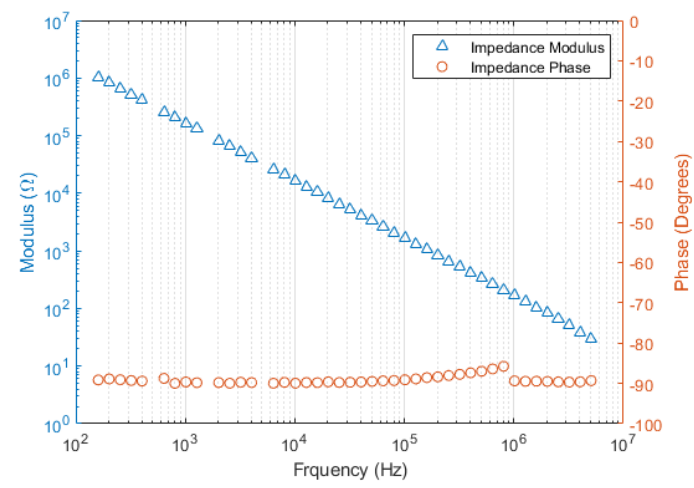

Figure 3. Complex impedance measurement (zero DC bias).

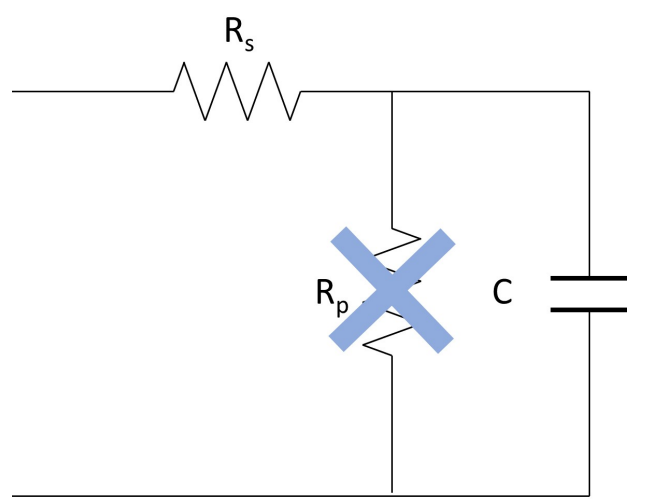

Figure 4. Resulting equivalent circuit without DC bias current.

Then, the swipe is not performed in frequency but in DC bias, going from 1 to $90 \mathrm{~mA}$. It is important to notice that when the impedance measured values are under $10 \Omega$, the SOLARTRON 1260 analyzer can only measure up to $100 \mathrm{kHz}$; beyond this frequency, the impedance measurement has a high variation, showing peaks, and cannot be trusted. The results depicted in Figure 5 obtained by changing the polarization current of the LED imply that the LED has a purely resistive (zero phase) behavior and corresponds to the sum of the resistances $R_{p}$ and $R_{s}$ as shown in Figure 6. 


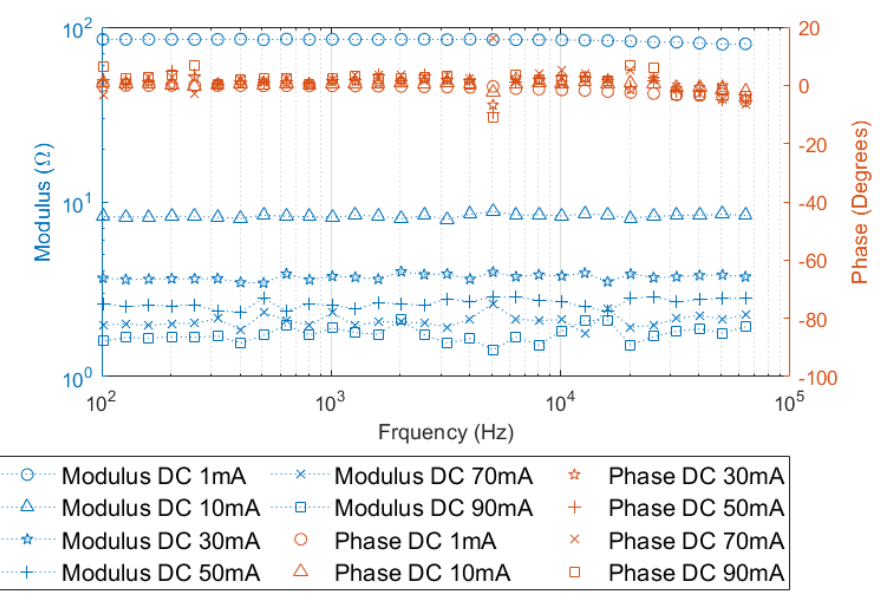

Figure 5. Complex impedance measurement (current DC bias from 1 to $90 \mathrm{~mA}$ ).

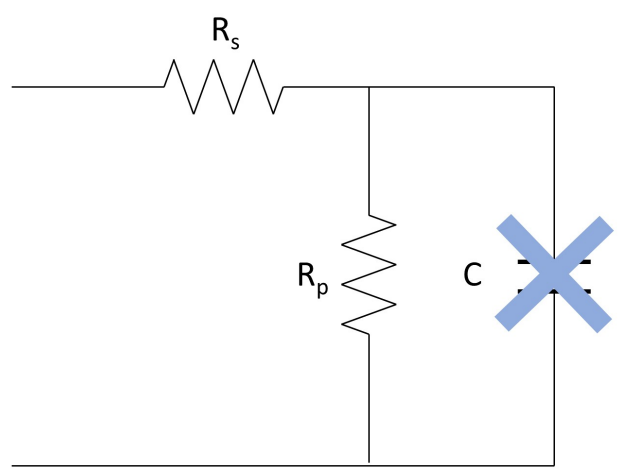

Figure 6. Resulting equivalent circuit with DC bias current.

It should be mentioned that $R_{p}$ is inversely proportional to the polarization DC bias current (Figure 7). Most importantly, it can be concluded that $R_{p}$ is equal to a division between a constant voltage value $(83.57 \mathrm{mV})$ over the LED polarization current denoted by $I_{\mathrm{DC}}$. Actually, the value of $R_{p}$ can be defined as $\frac{\eta K T}{q}$, where $\eta$ is an experimental coefficient that depends on the LED, $K$ is the Boltzmann constant, $T$ is the junction temperature, and $q$ is the electron charge.

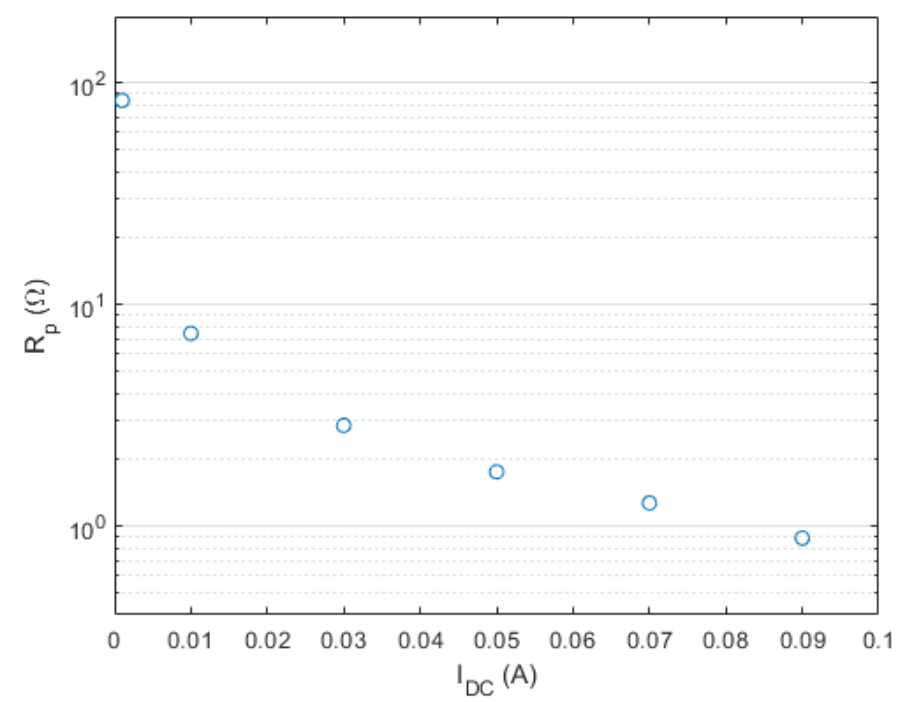

Figure 7. Value of $R_{p}$ obtained at different current bias using an impedance analyzer. 
The traditional measurement methodology, in this case, using a SOLARTRON 1260 analyzer for determining the electrical equivalent of the LEDs, e.g., the considered LED LXHL-BW02 is limited to frequency values below $100 \mathrm{kHz}$. Furthermore, the maximum value of polarization current that can be introduced to the LED is equal to $80 \mathrm{~mA}$. Consequently, the equivalent circuit's resistance and capacitance cannot be determined in a reliable fashion for characterizing the LEDs employed in VLC, which operates at high power and high modulation frequencies. In the following, we propose a novel methodology to characterize the LEDs' electrical response subject to high current variation at high modulation frequencies, in the $\mathrm{MHz}$ range.

\subsection{Impedance Measurement of the LED Using a Lock-In Amplifier}

We first propose an alternative methodology for determining the electrical response of the LEDs based on the concept of a bias-tee (Bias-T) diplexer. Basically, the Bias-T allows us to configure the DC polarization point of the LED, around which the alternating current (AC) varies without affecting the performance of the DC current source and the function generator. Thus, we propose a measurement circuit, as is shown in Figure 8. In this case, notice that the current source $I_{p o l}$ controls the LED polarization point.

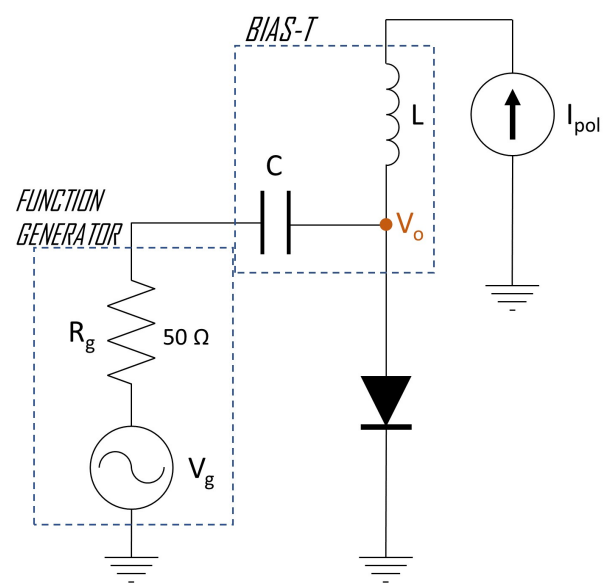

Figure 8. Circuit for the impedance measurement of the LED based on the Bias-T configuration.

By replacing the LED with the simplified equivalent circuit (see Figure 1), the proposed measurement circuit in the small signal is equivalent to the circuit shown in Figure 9. As the impedance of the LED decreases while the polarization current increases, in practice, for medium-high power LEDs (typically employed in VLC applications), the value of this impedance is below $1 \Omega$. As a consequence, the signal measured in the LED is extremely weak. Notice that since most of the signal intended for the LED is in fact in the generator inner resistance $\mathrm{Rg}$, measuring the voltage $V_{o}$ with an oscilloscope probe makes it such that other non-desired signals appear with power levels greater than the desired signal, and therefore, hampering to obtain a correct measurement of the electric response of the LED.

Then, we propose the use of a lock-in amplifier to measure the voltage $V_{o}$ in the above circuit. The lock-in is mostly the combination of a low-pass filter and an analog multiplier. The multiplier takes the test signal and the inner signal of the lock-in and multiplies them, then, by filtering the resulting multiplied signal, a DC equivalent value can be obtained. The low pass filter plays a key role because it has to remove or at least attenuate undesired signals (e.g., noise) while maintaining the desired signal intact. That means that the low pass filter must have an extremely narrow bandpass. Therefore, the measured signal must have the same frequency as the reference signal. 


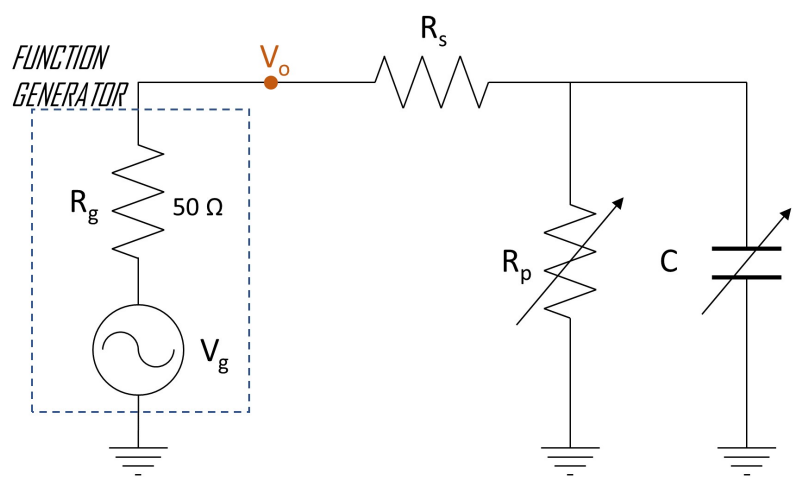

Figure 9. Resulting circuit of the Bias-T configuration replacing the LED with its equivalent circuit.

The Zurich elements lock-in amplifier HF2LI is used for this research since it provides a wide operating bandwidth (up to $50 \mathrm{MHz}$ ) [28]. At first, the lock-in amplifier must be calibrated to obtain feasible measures, and this is achieved by substituting the LED (see Figures 8 and 9) with a short resistance value. More precisely, resistance values of $0.25 \Omega$, $0.5 \Omega$ and $1 \Omega$ are considered in this work. Those resistances are close to the ones expected from the LED equivalent circuit and allow the identification of the lock-in typical voltage values at the different know resistance values.

For the LED LXHL-BW02 considered in this work, the measures collected using the lock-in amplifier proposed method are depicted in Figure 10. For these measurements, the current polarization shift used to obtain the impedance of the LED varies from $8.6 \mathrm{~mA}$ to $210 \mathrm{~mA}$. It is worth recalling that $210 \mathrm{~mA}$ corresponds to an average operation forward current of the LED LXHL-BW02. It can be seen that, as occurs with the measurements obtained with the SOLARTRON 1260 analyzer, the value of the resistance $R_{p}$ is inversely proportional to the polarization current. For current values over $160 \mathrm{~mA}$, the value of the series resistance is $0.83 \Omega$, and therefore, the value of the resistance $R_{p}$ corresponds to a very small value. In comparison, the obtained resistance value is like the one obtained using the impedance analyzer.

Thus, for signals with a frequency beyond $1 \mathrm{MHz}$, i.e., for a modulation bandwidth greater than this value in VLC, the response of the LED is determined not only by the resistance factor, but the capacitance also affects its response. Moreover, the phase obtained in the lock-in amplifier is not zero. The equation that models the resulting capacitance value is the following:

$$
C=\frac{\sqrt{R_{p}^{2}-|Z(\omega)|^{2}}}{R_{p} \cdot \omega \cdot|Z(\omega)|}
$$

where $|Z(\omega)|$ is the value of the measured impedance obtained through the lock-in amplifier at frequency $\omega$. In this case, the capacitance response of the LED LXHL-BW02 is appreciable for frequencies below $1 \mathrm{MHz}$. Notice that the phase measured at the lock-in amplifier corresponds to a non-zero value.

The modulus of the impedance is measured, polarizing the LED with a current of $16 \mathrm{~mA}$ at a frequency of $10 \mathrm{MHz}$. Even when the polarization current affects the capacitance value, as the current values are relatively small for this experiment, it is assumed that the capacitance value will remain constant and approximately equal to $5.02 \mathrm{nF}$ within the employed current range. 


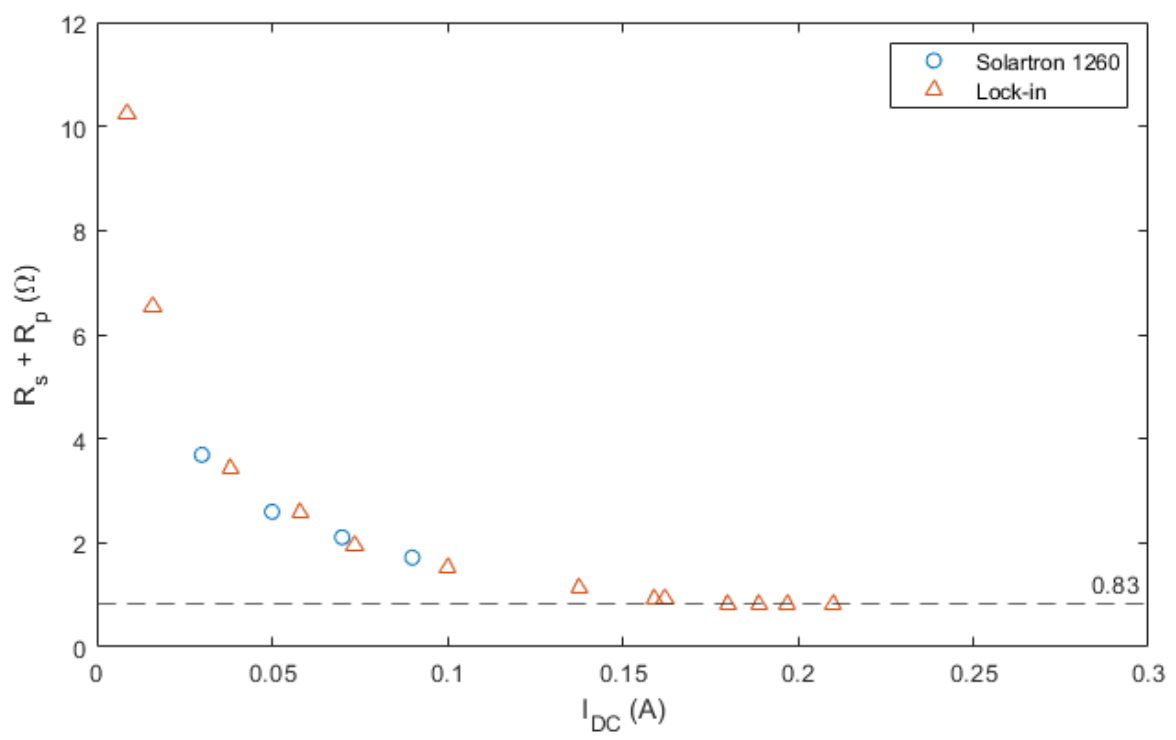

Figure 10. Value of $R_{s}+R_{p}$ obtained at different current bias using a look-in amplifier.

\section{Resulting Electrical Equivalent Circuit}

The obtained results are validated using software simulations. In this sense, the electronic circuits depicted in Figures 8 and 9 are simulated using LTSPICE software. It is worth remarking that the selection of the LXHL-BW02 is significant since the libraries of this specific LED are available in LTS-SPICE, which does not occur for most of the medium-high power LEDs. Therefore, the results obtained through simulations can be compared with the resulting equivalent circuit obtained with the lock-in amplifier proposed methodology as shown in Figure 11.

.param corriente $=210 \mathrm{~m} \mathrm{Rp}=4^{x} 0.0162 /$ corriente
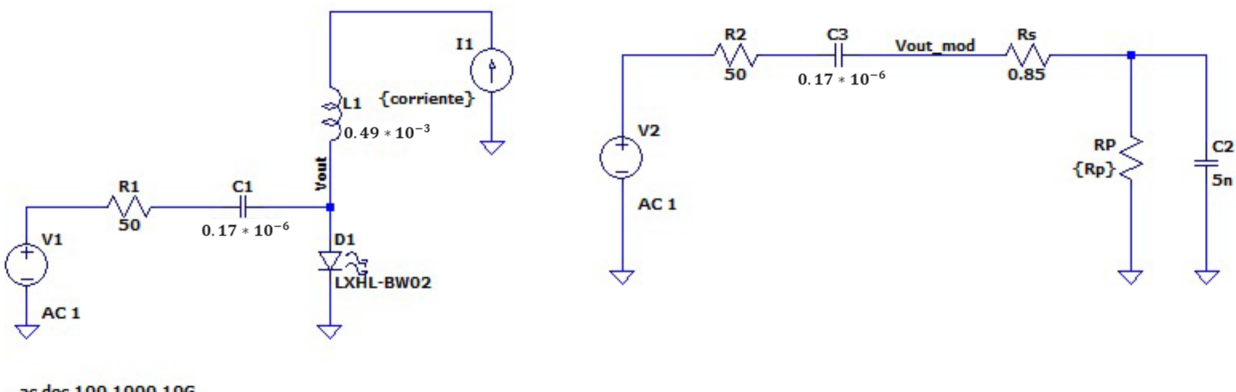

Figure 11. Simulated electrical circuits (comparison of LTSPICE model and equivalent circuit).

Table 1. Measuring parameters.

\begin{tabular}{cc}
\hline Parameter & Value \\
\hline Analyzed LED & Luxeon LXHL-BW02 [27] \\
\hline Typical (Maximum) input current & $210(350) \mathrm{mA}$ \\
\hline Typical luminous flux & $45 \mathrm{~lm}$ \\
\hline
\end{tabular}

The simulations consider a frequency sweep from $1 \mathrm{kHz}$ to $1 \mathrm{GHz}$. Figure 12 (polarization current of $210 \mathrm{~mA}$ ) and Figure 13 (polarization current of $16 \mathrm{~mA}$ ) compare the frequency response obtained for both $V_{o} u t$ and $V_{o} u t m o d$. These current values were se- 
lected following the LED parameters stated in Table 1. Furthermore, it is worth noticing that analyzing Figure 11, the following equation can be determined:

$$
\frac{V_{0}(s)}{V_{i}(s)}=G_{0} \cdot \frac{1+\frac{s}{\omega_{c}}}{1+\frac{s}{\omega_{p}}}
$$

where

$$
\begin{gathered}
G_{0}=\frac{R_{s}+R_{p}}{R_{g}+R_{s}+R_{p}} \\
\omega_{c}=\frac{R_{s}+R_{p}}{R_{s} \cdot R_{p} \cdot C}
\end{gathered}
$$

and

$$
\omega_{p}=\frac{R_{g}+R_{s}+R_{p}}{R_{p} \cdot\left(R_{g}+R_{s}\right) \cdot C}
$$

Checking these equations and also the simulation results, we can conclude that, in frequency, the signal generator output resistance creates a zero very close to the righthand of the pole. Because of the influence of this pole, the gain suffers a significant reduction, remaining constant at the frequency of the zero.

Figures 12 and 13 show the measured frequency response of the considered highpower LEDs. It can be seen that the available modulation bandwidth is constant from $100 \mathrm{kHz}$ to $10 \mathrm{MHz}$, approximately. This bandwidth is large enough for most of the applications in 6G. Recall that the use of signal processing techniques increases the spectral efficiency of this bandwidth. However, notice that the phase is clearly not linear within the constant bandwidth. Consequently, the implementation of signal processing techniques requires to implement a pre-distortion or an equalizer stage to exploit the modulation bandwidth of the high-power LEDs. In this sense, the methodology proposed in this work allows us to determine the electronic equivalent of the high-power LEDs, which is the first step for implementing pre-distortion or equalizer stages that overcome the non-linear or frequency selective response of the high-power LEDs.

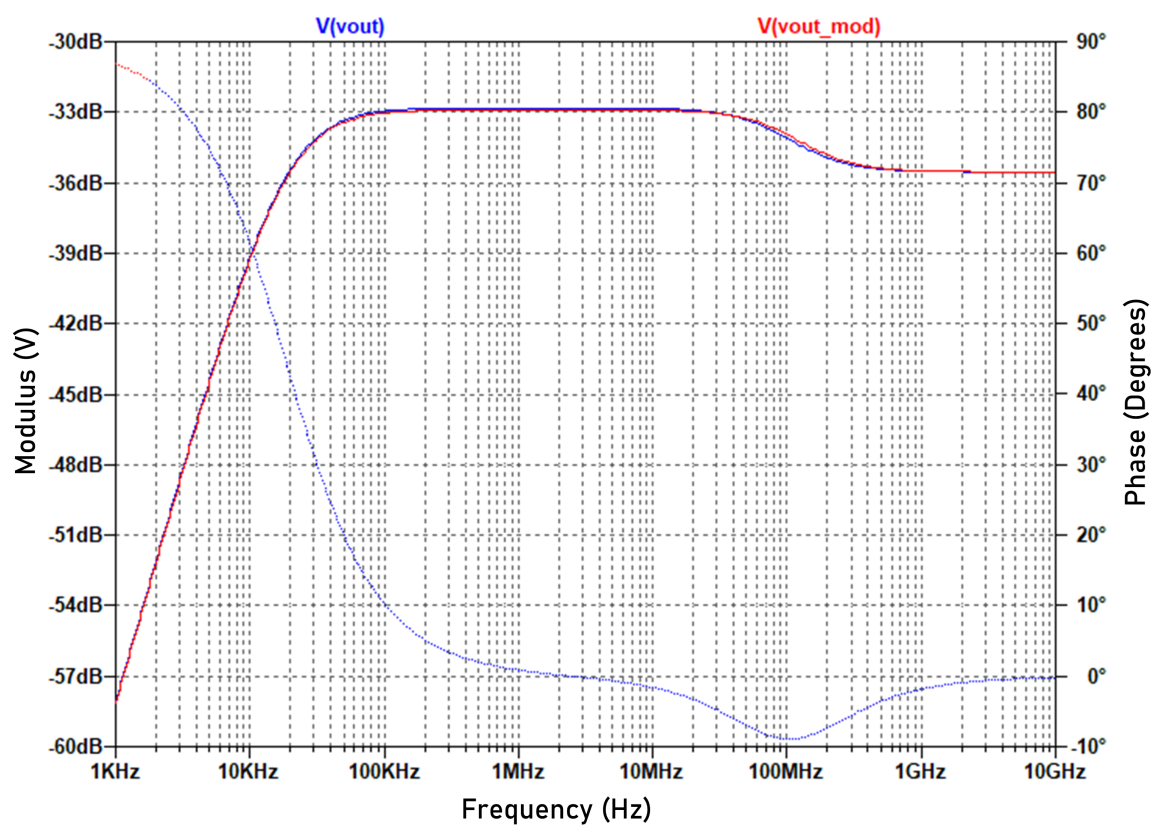

Figure 12. Bode response of the LTSPICE model and equivalent circuit simulation of the LED (polarization current of $210 \mathrm{~mA}$ ). 


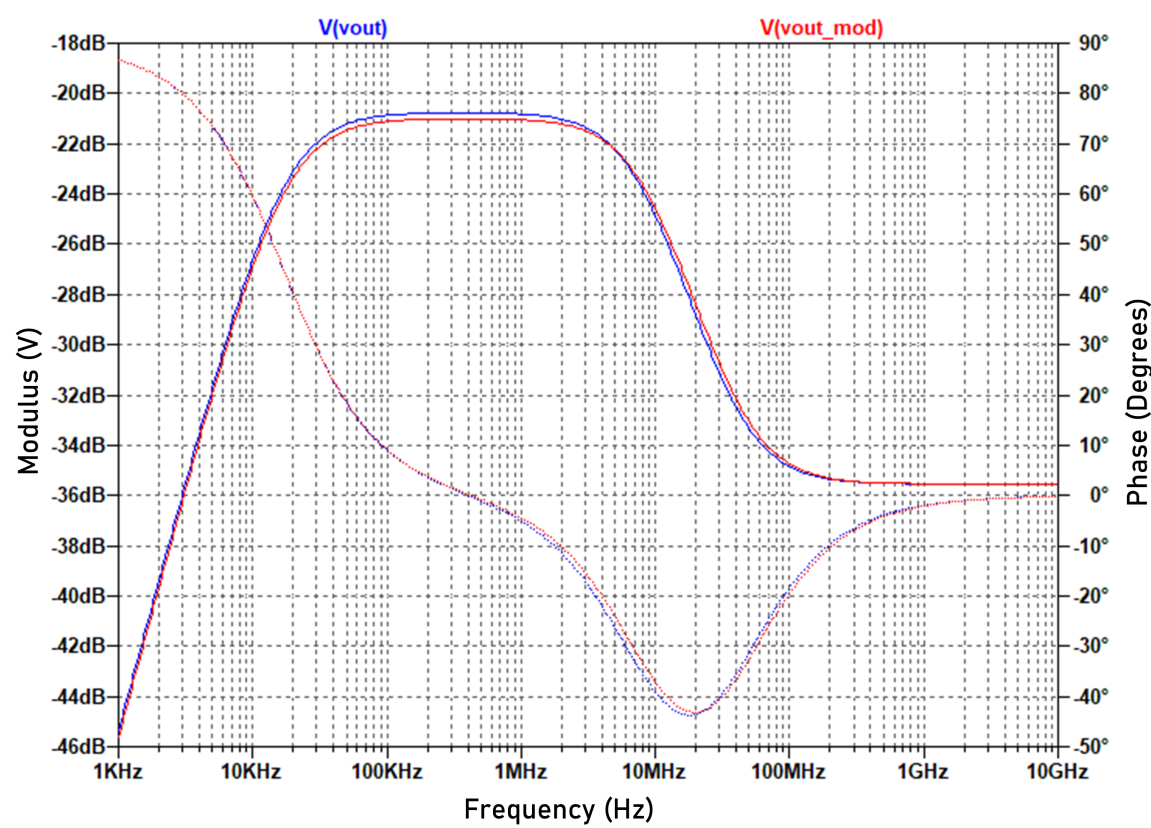

Figure 13. Bode response of the LTSPICE model and equivalent circuit simulation of the LED (polarization current of $16 \mathrm{~mA}$ ).

\section{Conclusions}

High-power LEDs have their equivalent impedance decreased during the current rise. As these components operate with high currents, their equivalent impedance is around a few ohms, resulting in several hurdles in order to measure them by using "classical" measurement devices, such as LCR meters or impedance analyzers.

Traditional impedance analyzers have limitations in both the measurement bandwidth and the current that can be supplied to the measured component (load). Notice that these analyzers are mainly conceived to measure passive components or components with low polarization current.

A novel alternative method to measure the dynamic behavior of high-power LEDs and then define their equivalent circuit is proposed. The use of a Bias-T circuit for determining the LED equivalent circuit is proposed. However, it is shown that the use of a Bias-T to polarize the LED is not the most convenient approach, and it is because of a large part of the signal that falls into the Bias-T internal impedance.

By using a lock-in amplifier, we have enough room to characterize the capacitance and resistance of the LED equivalent circuit, as impedance measurements can be performed up to $50 \mathrm{MHz}$.

The resulting equivalent circuit allows simulating the electrical behavior of any highpower LED. In the case of this paper, the response of the resulting equivalent circuit is compared with the spice model of the LED LXHL-BW02, obtaining an equal frequency behavior (Figures 12 and 13). As most of the high-power white LEDs do not have a frequency spice model, the equivalent circuit is the only way to analyze the frequency response of those LEDs.

By increasing the LEDs' polarization current, the value of Rp decreases, and then, the electrical bandwidth of LEDs is increased (Figure 5). Therefore, as the LED optical bandwidth does not reach such frequencies, it can be said that the LEDs bandwidth limitation is not electrical but optical. It may imply that using an equalization circuit on the transmitter side is not the optimal approach. A blue optical filter can increase the bandwidth of the white LED, although it considerably reduces the optical power received [29]. In addition, the use of advanced modulation techniques for high data rate VLC transmission could improve the efficiency of the channel [30]. 


\begin{abstract}
Author Contributions: Conceptualization, J.C.T.Z. and J.S.B.P.; formal analysis, C.I.d.V.; investigation, J.C.T.Z. and J.S.B.P.; methodology, J.C.T.Z.; software, J.C.T.Z. and J.S.B.P.; supervision, J.M.S.P. and I.M.-S.; validation, M.M.C.; visualization, M.M.C. and J.S.B.P.; writing—original draft, J.C.T.Z.; writing-review and editing, J.M.S.P., M.M.C. and J.S.B.P. All authors have read and agreed to the published version of the manuscript.
\end{abstract}

Funding: This research was co-financed by Comunidad de Madrid and the FSE/FEDER Program under grant SINFOTON2-CM (S2018/NMT-4326), the Madrid Government (Comunidad de Madrid) under the Multiannual Agreement with UC3M in the line of "Fostering Young Doctors Research" (GEOVEOLUZ-CM-UC3M), and in the context of the V PRICIT (Regional Programme of Research and Technological Innovation, and the Ministerio de Ciencia e Innovación and Agencia Estatal de Investigación (PID2019-109072RB-C31) and under the CDTI (Centre for the Development of Industrial Technology, Ministerio de Ciencia e Innovación) throughthe European Regional Development Fund (ERDF) EXP 00119337/IDI-2019029.

Conflicts of Interest: The authors declare no conflict of interest.

\title{
References
}

1. Willardson, R.K.; Weber, E.R.; Stringfellow, G.B.; Craford, M.G. High Brightness Light Emitting Diodes; Elsevier Science: Amsterdam, The Netherlands, 1997.

2. Nakamura, S.; Mukai, T.; Senoh, M. Candela-class high-brightness InGaN/AlGaN double-heterostructure blue-light-emitting diodes. Appl. Phys. Lett. 1994, 64, 1687-1689. [CrossRef]

3. Narukawa, Y.; Narita, J.; Sakamoto, T.; Deguchi, K.; Yamada, T.; Mukai, T. Ultra-High Efficiency White Light Emitting Diodes. Jpn. J. Appl. Phys. 2006, 45, L1084-L1086. [CrossRef]

4. Haas, H.; Yin, L.; Wang, Y.; Chen, C. What is LiFi? J. Light. Technol. 2016, 34, 1533-1544. [CrossRef]

5. Jovicic, A.; Li, J.; Richardson, T. Visible light communication: Opportunities, challenges and the path to market. IEEE Commun. Mag. 2013, 51, 26-32. [CrossRef]

6. Almadani, Y.; Plets, D.; Bastiaens, S.; Joseph, W.; Ijaz, M.; Ghassemlooy, Z.; Rajbhandari, S. Visible Light Communications for Industrial Applications-Challenges and Potentials. Electronics 2020, 9, 2157. [CrossRef]

7. Céspedes, M.M.; Guzmán, B.G.; Jiménez, V.P.G. Lights and Shadows: A Comprehensive Survey on Cooperative and Precoding Schemes to Overcome LOS Blockage and Interference in Indoor VLC. Sensors 2021, 21, 861. [CrossRef]

8. Wagh, P.; Patil, S. Transmission of biomedical signal using VLC based system in hospitals. In Proceedings of the 2019 3rd International Conference on Trends in Electronics and Informatics (ICOEI), Tirunelveli, India, 23-25 April 2019 ; pp. 110-112. [CrossRef]

9. Iqrar, A.; Karvonen, H.; Kumpuniemi, T.; Katz, M. Wireless Communications for the Hospital of the Future: Requirements, Challenges and Solutions. Int. J. Wirel. Inf. Netw. 2020, 27, 4-17.

10. Barman, A.D.; Halder, A. Indoor visible light communication with smart lighting technology. In Proceedings of the Smart Photonic and Optoelectronic Integrated Circuits XIX, San Francisco, CA, USA, 31 January-2 February 2017; Eldada, L.A., Lee, E.H., He, S., Eds.; International Society for Optics and Photonics: Bellingham, WA, USA, 2017; Volume 10107, pp. 112-122.

11. Huynh, P.; Yoo, M. VLC-Based Positioning System for an Indoor Environment Using an Image Sensor and an Accelerometer Sensor. Sensors 2016, 16, 783. [CrossRef]

12. Lv, H.; Feng, L.; Yang, A.; Guo, P.; Huang, H.; Chen, S. High Accuracy VLC Indoor Positioning System with Differential Detection. IEEE Photonics J. 2017, 9, 1-13. [CrossRef]

13. Torres, J.C.; Montes, A.; Mendoza, S.L.; Fernández, P.R.; Betancourt, J.S.; Escandell, L.; del Valle, C.I.; Sánchez-Pena, J.M. A Low-Cost Visible Light Positioning System for Indoor Positioning. Sensors 2020, 20, 5145. [CrossRef]

14. Tsonev, D.; Chun, H.; Rajbhandari, S.; McKendry, J.J.D.; Videv, S.; Gu, E.; Haji, M.; Watson, S.; Kelly, A.E.; Faulkner, G.; et al. A 3-Gb/s Single-LED OFDM-Based Wireless VLC Link Using a Gallium Nitride $\mu$ LED. IEEE Photonics Technol. Lett. 2014, 26, 637-640. [CrossRef]

15. Yapici, Y.; Guvenc, I. NOMA for VLC Downlink Transmission with Random Receiver Orientation. IEEE Trans. Commun. 2019, 67, 5558-5573. [CrossRef]

16. Obeed, M.; Salhab, A.M.; Alouini, M.S.; Zummo, S.A. On Optimizing VLC Networks for Downlink Multi-User Transmission: A Survey. IEEE Commun. Surv. Tutor. 2019, 21, 2947-2976. [CrossRef]

17. Viñals, R.; Muñoz, O.; Agustín, A.; Vidal, J. Multi-User Precoder Designs for RGB Visible Light Communication Systems. Sensors 2020, 20, 6836. [CrossRef]

18. Little, T.; Rahaim, M.; Abdalla, I.; Lam, E.; Mcallister, R.; Vegni, A.M. A multi-cell lighting testbed for VLC and VLP. In Proceedings of the 2018 Global LIFI Congress (GLC), Paris, France, 8-9 February 2018; pp. 1-6. [CrossRef]

19. Fortes, M.; González, O. Testbed for Experimental Characterization of Indoor Visible Light Communication Channels. Electronics 2021, 10, 1365. [CrossRef]

20. Chi, N.; Zhou, Y.; Wei, Y.; Hu, F. Visible Light Communication in 6G: Advances, Challenges, and Prospects. IEEE Veh. Technol. Mag. 2020, 15, 93-102. [CrossRef] 
21. Alsharif, M.H.; Kelechi, A.H.; Albreem, M.A.; Chaudhry, S.A.; Zia, M.S.; Kim, S. Sixth Generation (6G) Wireless Networks: Vision, Research Activities, Challenges and Potential Solutions. Symmetry 2020, 12, 676. [CrossRef]

22. Chen, C.; Deng, X.; Yang, Y.; Du, P.; Yang, H.; Zhao, L. LED Nonlinearity Estimation and Compensation in VLC Systems Using Probabilistic Bayesian Learning. Appl. Sci. 2019, 9, 2711. [CrossRef]

23. Li, X.; Ghassemlooy, Z.; Zvánovec, S.; Haigh, P.A. A 40 Mb/s VLC System Reusing an Existing Large LED Panel in an Indoor Office Environment. Sensors 2021, 21, 1697. [CrossRef] [PubMed]

24. Zissis, G.; Bertoldi, P.; Ribeiro Serrenho, T. Update on the Status of LED-Lighting World Market since 2018; Publications Office of the European Union: Luxembourg, 2021. [CrossRef]

25. Li, X.; Ghassemlooy, Z.; Zvanovec, S.; Alves, L.N. An Equivalent Circuit Model of a Commercial LED With an ESD Protection Component for VLC. IEEE Photonics Technol. Lett. 2021, 33, 777-779. [CrossRef]

26. Ricky Lee, S.W.; Jeffery, C.C.L. From LED to Solid State Lighting: Principles, Materials, Packaging, Characterization, and Applications; Wiley: Hoboken, NJ, USA, 2021.

27. Power Light Source LUXEON ${ }^{\circledR}$ Emitter. Available online: https://www.luxeonstar.com/assets/downloads/ds25.pdf (accessed on 17 January 2022).

28. Zurich Instruments. HF2LI $50 \mathrm{MHz}$ Lock-in Amplifier. Available online: https://www.zhinst.com/others/en/products/hf2lilock-in-amplifier (accessed on 17 January 2022).

29. Sung, J.Y.; Chow, C.W.; Yeh, C.H. Is blue optical filter necessary in high speed phosphor-based white light LED visible light communications? Opt. Express 2014, 22, 20646-20651. [CrossRef] [PubMed]

30. Chun, H.; Rajbhandari, S.; Faulkner, G.; Tsonev, D.; Haas, H.; O’Brien, D. Demonstration of a Bi-directional visible light communication with an overall sum-rate of $110 \mathrm{Mb} / \mathrm{s}$ using LEDs as emitter and detector. In Proceedings of the $2014 \mathrm{IEEE}$ Photonics Conference, San Diego, CA, USA, 12-16 October 2014; pp. 132-133. [CrossRef] 